\title{
versants
}

\section{Henri Roorda, encore méconnu}

\author{
Daniel MagGeTti \\ Université de Lausanne
}

\begin{abstract}
Henri Roorda est à la fois un auteur très apprécié par un cercle d'amateurs, et un écrivain largement méconnu. Ce cahier souhaite donner quelques pistes pour découvrir une œuvre variée à l'humour souvent décapant.

Keywords: Henri Roorda, chronique, association, Michel Froidevaux, pédagogie.
\end{abstract}

En Suisse romande, Henri Roorda (I870-1925) est à la fois une référence presque classique, et un écrivain mal connu. Par la place qu'il a prise dans la critique du système scolaire, grâce en particulier à son célèbre Le Pédagogue n'aime pas les enfants, paru en 1917 à l'enseigne des Cahiers vaudois et qui a fait date dans l'histoire de la pédagogie, Roorda jouit d'un statut intellectuel indiscuté. Sur le plan strictement littéraire, en revanche, sa reconnaissance a été plus tardive et demeure partielle. Les circonstances biographiques et éditoriales expliquent cet état des choses. De son temps et dans le contexte vaudois, Roorda a été un auteur marginal, à cause de ses idées libertaires, de sa non-appartenance aux cercles littéraires en vue, de sa pratique d'écriture privilégiant des genres et des formes peu légitimes, le théâtre et surtout la chronique journalistique, dont la labilité n'a pas été entièrement contrecarrée par les sélections qu'il en a réalisé en volume de son vivant. La fin de vie dramatique de Roorda, sur laquelle son autre œuvre la plus marquante, Mon suicide (1925), lève le voile, a elle aussi eu des effets sur sa réception, d'une part parce qu'elle lui a valu de facto une réputation plus que singulière aux yeux de ses contemporains, et d'autre part parce qu'il a de lui-même, avant sa mort, détruit les traces matérielles de son travail d'écrivain, de sa réception immédiate et de ses retombées. Il a fallu attendre 1970 pour que s'amorce une (re)découverte, avec la publication des deux volumes d'Euvres complètes aux Éditions L'Âge d'homme. Ces œuvres complètes n'en sont toutefois pas : n'y figure pas une vaste partie de la production de chroniqueur de Roorda, restée enfouie dans les quotidiens qui l'avaient accueillie. Par ailleurs, l'accompagnement critique plus que réduit de cette édition n'offre pas au lecteur les éléments de contextualisation et de mise en perspective qui sont pourtant indispensables pour prendre pleinement la mesure de la stature de l'écrivain.

Depuis les années 1980, et de manière plus soutenue au fil des vingt dernières années, les textes de Roorda ont connu une diffusion sans précédent, que ce soit au théâtre, grâce à divers spectacles et mise en scène, ou dans le 
domaine éditorial, avec une série de publications - rééditions diverses, exhumations de chroniques, traductions en plusieurs langues. La plupart de ces heureuses initiatives sont dues à l'engagement de l'Association des Amis de Henri Roorda, créée à Lausanne en 2003, dont le but est précisément la valorisation et la diffusion de l'œuvre et de la pensée de l'écrivain. Parmi ses réalisations les plus significatives, une exposition au Musée historique de Lausanne, en 2009, dont le catalogue, également intitulé Henri Roorda et l'humour zèbre, constitue à ce jour le seul ouvrage de référence rassemblant des études et des analyses du parcours et de la production de l'auteur. Le site de l'association, http://henri-roorda.org, fait du reste référence pour tout renseignement biographique et bibliographique au sujet de l'écrivain, et nous y renvoyons à notre tour le lecteur.

Comme nous venons de le rappeler, la voix de Roorda a donc recommencé à circuler, son ton et son humour ont pu, à diverses reprises, produire à nouveau leur effet, en faisant sourire et en déstabilisant à la fois. Le constat est heureux, mais en parallèle, on ne peut manquer de relever que du point de vue de la critique, le chemin à faire est encore long. Ce dossier dans Versants voudrait être une contribution à un mouvement dont on espère qu'il ne cessera de prendre de l'ampleur. On y retrouvera le chroniqueur, l'épistolier, le pédagogue, tantôt à la première personne, tantôt abordé par ses textes, en français ou en traduction.

Ce numéro de Versants était tout juste terminé que paraissait aux Éditions la Baconnière à Genève un recueil de chroniques non encore reprises en volume, préparé par Jonathan Wenger et intitulé Intelligence à louer. Une coïncidence réjouissante! Y figure notamment le texte « Les vertus universelles", reproduit dans ce numéro.

Nous dédions cet ensemble à la mémoire de Michel Froidevaux, décédé en novembre 2020. L'éditeur, collectionneur et galeriste lausannois, fondateur et président de l'Association des Amis de Henri Roorda, en a été l'infatigable promoteur. Sa disparition est une grande perte : c'est à lui qu'on doit en premier lieu d'avoir amorcé et soutenu une redécouverte dont tous les lecteurs de Roorda-Balthasar lui sont très reconnaissants. On ne l'oubliera pas. 